\title{
The molecular clouds in the environs of the supernova remnants G349.7+0.2 and G18.8+0.3
}

\author{
G. Dubner ${ }^{\star \star \star}$, E. Giacani, E. Reynoso, and S. Parón ${ }^{\star \star \star}$ \\ Instituto de Astronomía y Física del Espacio (IAFE), CC 67, Suc. 28, 1428 Buenos Aires, Argentina \\ e-mail: gdubner@iafe.uba.ar
}

Received 20 May 2004 / Accepted 25 June 2004

\begin{abstract}
We present the results of a new high-resolution study of the molecular gas associated with the supernova remnants (SNRs) G349.7+0.2 and G18.8+0.3. The observations were performed with the SEST telescope in the ${ }^{12} \mathrm{CO} J=1-0,2-1$ and 3-2 lines (beams of 45", 23" and 15", respectively). The present observations have provided, for the two SNRs, new evidence in support of the existence of physical interaction between the SN shocks and the adjoining molecular clouds. In the case of G349.7+0.2, the new observations revealed for the first time the internal structure of the shocked cloud, as well as the kinematical consequences of the impact of the SNR shock on the molecular cloud. From these observations we were able to constrain the conditions of the pre-shocked gas. The molecular cloud associated with G349.7+0.2, centered near $v_{\mathrm{LSR}}=+16.2 \mathrm{~km} \mathrm{~s}^{-1}$, has a linear size of about $7 \mathrm{pc}$, a mass of $\sim 10^{4} M_{\odot}$ and a volume density of $\sim 10^{3} \mathrm{~cm}^{-3}$. The high line ratios derived are indicative of the existence of shocks in the cloud. From the asymmetries observed in the line shapes we propose that the SN shock cloud is running into the denser part of the cloud and has probably begun to disrupt it, pushing the eastern component clumps away from us, and the western fragments toward us. After comparing our estimates of the column density of the intervening gas with similar calculations based on ASCA X-rays spectral fitting we conclude that the best way to make these results compatible is by assuming that the associated cloud is placed behind G349.7+0.2 along the line of sight, and the SNR/molecular cloud encounter is taking place on the far side of the SNR. This model also provides a natural explanation for the lack of strong $\mathrm{X}$-ray absorption in the central region of G349.7+0.2. Evaporation of part of the associated cloud must be responsible for the central X-ray emission. The comparison with IRAS infrared data provides additional support for the hypothesis of SNR/cloud physical interaction. From the study of the molecular gas in the neighborhood of the five $\mathrm{OH}(1720 \mathrm{MHz})$ masers detected in G349.7+0.2 we find that in three cases the maser peak velocity coincides with the local CO peak velocity, while in the remaining two cases the maser peak velocity agrees with a secondary, blended $\mathrm{CO}$ component. We conclude that the masers are excited at the sites where a non-dissociative C-type shock, locally transverse to the line of sight (or forming a large angle with it), hits a denser molecular clump. For the SNR G18.8+0.3, the new higher resolution observations have revealed excellent morphological agreement between one of the cloud components and the SNR shock front towards the eastern limb. The associated molecular mass is estimated to be $\sim 4.4 \times 10^{4} M_{\odot}$ and the cloud volume density $\sim 1200 \mathrm{~cm}^{-3}$. The analysis of the line ratios in this case revealed a maximum of $R_{2-1 / 1-0}=1.25$ at a position that exactly matches an indentation in the radio continuum emission in the remnant's shell, providing additional evidence of $\mathrm{SNR} /$ molecular cloud interaction.
\end{abstract}

Key words. ISM: molecules - ISM: clouds - ISM: supernova remnants - radio lines: ISM

\section{Introduction}

Supernova explosions are among the basic processes which determine and control the physical and chemical state of the interstellar medium (ISM). The shock waves generated by supernova remnants (SNRs) can accelerate, compress, heat, fragment or even destroy surrounding interstellar clouds. They can enhance or reduce abundances with respect to quiescent cloud conditions of different molecular species. Observations of molecular clouds adjacent to SNRs provide information

^ Visiting Astronomer, European Southern Observatory.

$\star \star$ G.D., E.G., and E.R. are members of the Carrera del Investigador Científico of CONICET, Argentina.

$\star \star \star$ Doctoral Fellow of CONICET, Argentina. essential to the understanding of the physics involved in these processes.

There are still very few cases where the mutual interaction between a supernova shock and an external cloud has been well proven and investigated (see for example Seta et al. 1998 for a compilation of detections). Morphological signatures are often invoked to suggest a physical association between an SNR and a molecular cloud (e.g., Tatematsu et al. 1987; Dubner et al. 1999). A powerful indication of SNR-molecular cloud interaction is the presence of $\mathrm{OH}(1720 \mathrm{MHz})$ shock-excited masers. In effect, observations and theory support the hypothesis that the action of SN shocks on clouds with molecular densities between $\sim 10^{3}$ and $\sim 10^{5} \mathrm{~cm}^{-3}$ and kinetic temperatures in the range $50-125 \mathrm{~K}$, can create a strong inversion in the population 


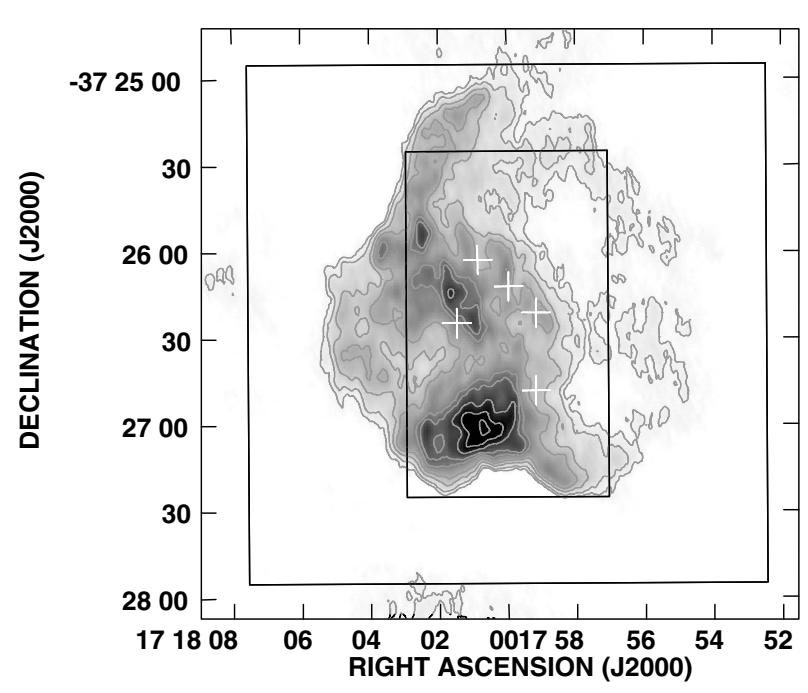

Fig. 1. VLA radio continuum image of G349.7+0.2 (from Brogan et al. 2000) displayed between 0 and $50 \mathrm{mJy} /$ beam. The crosses show the positions of the $\mathrm{OH}(1720 \mathrm{MHz})$ masers (from Frail et al. 1996). The rectangles show the areas observed in the different $\mathrm{CO}$ transitions. The outer one delimits the region observed with a 45" grid-spacing in ${ }^{12} \mathrm{CO} J=1-0$ and $2-1$ lines and the inner rectangle shows the area studied in the same lines and in ${ }^{12} \mathrm{CO} J=3-2$, with a 12 " grid-spacing.

originating the $1720 \mathrm{MHz}$ maser line (Elitzur 1976; Frail et al. 1996; Green et al. 1997; Koralesky et al. 1998; Lockett et al. 1999).

In this paper we present a new high-resolution study of the CO distribution towards two SNRs, G349.7+0.2 and G18.8+0.3 (also known as Kes 67), carried out in 1999 in the $J=1-0, J=2-1$ and $J=3-2$ transitions of the ${ }^{12} \mathrm{CO}$ using the $15 \mathrm{~m}$ Swedish-ESO Submillimetre Telescope (SEST), in La Silla (Chile). G349.7+0.2 has associated OH (1720 MHz) masers, and for G18.8+0.3 there is evidence of SNR-molecular cloud interaction, suggested on a morphological basis. In both SNRs, previous molecular and mid-infrared observations revealed the existence of neighboring molecular clouds (Dubner et al. 1999; Reynoso \& Mangum 2000; Lazendic et al. 2002).

G349.7+0.2 is a small (2.5 in diameter), irregular shell SNR with a bright emission ridge in the radio continuum running approximately in the NE-SW direction. Shaver et al. (1985) suggested that a strong, early interaction with a dense interstellar medium (ISM) was the cause of this synchrotron enhancement in the shell interior. Located at $22.4 \mathrm{kpc}$ (Frail et al. 1996), G349.7+0.2 is one of the brightest galactic SNRs in radio and in X-rays (Slane et al. 2002). Frail et al. (1996) detected five $\mathrm{OH}(1720 \mathrm{MHz})$ masers towards the center of the SNR, with LSR velocities in the interval $(+14.3,+16.9) \mathrm{km} \mathrm{s}^{-1}$, and Brogan et al. (2000) measured the magnetic field strength towards the three brightest masers. Based on CO $J=1-0$ data acquired with an angular resolution of 54", Reynoso \& Mangum (2000) detected a molecular cloud approximately encircled by the OH $1720 \mathrm{MHz}$ masers and at a similar systemic velocity. Lazendic et al. (2002) observed this SNR in several molecular lines, from radio to infrared, confirming the presence of the associated cloud. Figure 1 shows a radio continuum image of

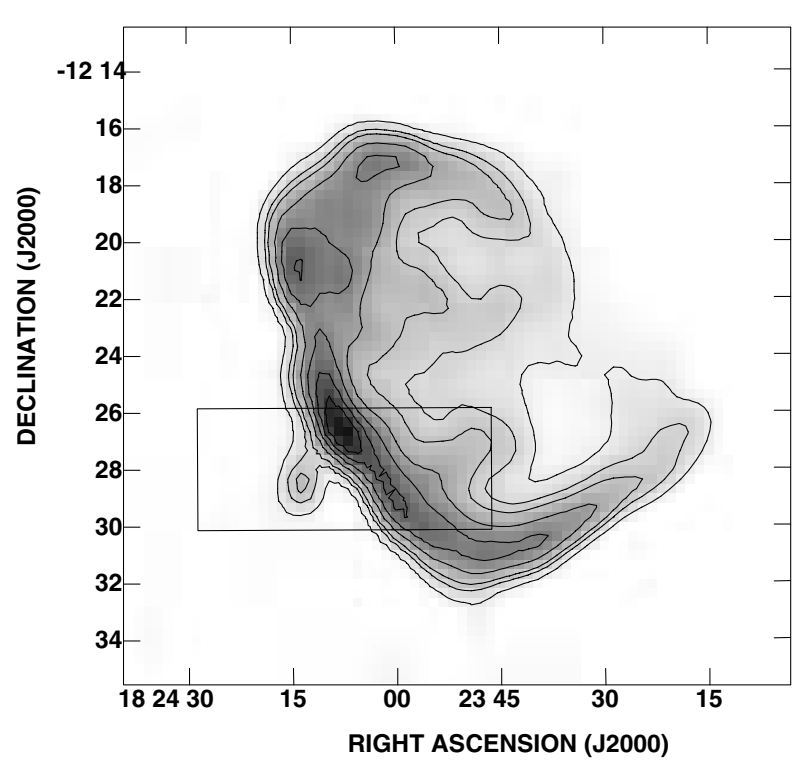

Fig. 2. Radio continuum emission associated with G18.8+0.3 (from Dubner et al. 1996). The greyscale varies between 20 and $800 \mathrm{mJy} /$ beam and the contours correspond to $80,150,200,300,400$, 600 and $800 \mathrm{mJy} /$ beam. The inner rectangle shows the area surveyed in the ${ }^{12} \mathrm{CO} J=1-0$ and $J=2-1$ lines.

the SNR G349.7+0.2 as obtained from a compilation of 18 and $20 \mathrm{~cm}$ VLA archive data (from Brogan et al. 2000), including the location of the areas surveyed in the $\mathrm{CO}$ lines in the present study (big and small rectangles). The crosses mark the location of the five $\mathrm{OH}(1720 \mathrm{MHz})$ masers (Frail et al. 1996).

In the case of G18.8+0.3, using NANTEN telescope data obtained with HPBW 2'8, Dubner et al. (1999) found an elongated molecular complex adjacent to the more flattened border of the SNR. The most remarkable radio-continuum/CO emission correspondence is observed for one of the clouds of the CO complex (named 3 in Dubner et al. 1999), located at the same place as a faint protrusion seen in the radio continuum. This cloud coincides in position with an IRAS point source whose spectrum is compatible with that of a protostar candidate (according to the criteria of Junkes et al. 1992), suggesting that the SNR/molecular cloud interaction might have triggered the formation of new stars at this location. Figure 2 shows the SNR G18.8+0.3 in radio continuum at $1.4 \mathrm{GHz}$ (Dubner et al. 1996) including the location of the area observed in the CO lines (rectangle).

\section{Observations}

\section{1. $S N R$ G349.7+0.2}

The observations of G349.7+0.2 were made over 4 nights from 1999 August 14 to August 17 using the 15m SwedishESO Submillimetre Telescope (SEST), in La Silla (Chile). We used SIS receivers to simultaneously observe the ${ }^{12} \mathrm{CO} J=1-$ $0(115.271 \mathrm{GHz})$ and ${ }^{12} \mathrm{CO} J=2-1$ lines $(230.538 \mathrm{GHz})$. Two Acousto-Optical spectrometers were used as back end: a narrow band high-resolution spectrometer (HRS) with 2000 channels, bandwidth $80 \mathrm{MHz}$, channel separation $41.7 \mathrm{kHz}$ (corresponding to $0.108 \mathrm{~km} \mathrm{~s}^{-1}$ and $0.054 \mathrm{~km} \mathrm{~s}^{-1}$ for the ${ }^{12} \mathrm{CO}$ 
Table 1. Observational parameters.

\begin{tabular}{|c|c|c|c|c|c|c|}
\hline Molecule & Transition & Beam & $\eta^{a}$ & $\overline{t^{b}(\mathrm{~s})}$ & $\operatorname{Noise}^{c}(\mathrm{~K})$ & $\Delta v^{d}\left(\mathrm{~km} \mathrm{~s}^{-1}\right)$ \\
\hline \multicolumn{7}{|c|}{$\mathrm{G} 18.8+0.3$} \\
\hline \multirow[t]{2}{*}{${ }^{12} \mathrm{CO}$} & $J=1-0$ & $45^{\prime \prime}$ & 0.70 & 60 & 0.20 & 0.32 (HRS-B) \\
\hline & & & & 60 & 0.18 & 1.80 (LR2-B) \\
\hline \multirow[t]{2}{*}{${ }^{12} \mathrm{CO}$} & $J=2-1$ & $23^{\prime \prime}$ & 0.50 & 60 & 0.30 & 0.05 (HRS-A) \\
\hline & & & & 60 & 0.07 & 0.91 (LR1-A) \\
\hline \multicolumn{7}{|c|}{ G349.7+0.2 } \\
\hline \multirow[t]{2}{*}{${ }^{12} \mathrm{CO}$} & $J=1-0$ & $45^{\prime \prime}$ & 0.70 & 60 & 0.11 & 0.32 (HRS-B) \\
\hline & & & & 60 & 0.18 & 1.80 (LR2-B) \\
\hline \multirow[t]{2}{*}{${ }^{12} \mathrm{CO}$} & $J=2-1$ & $23^{\prime \prime}$ & 0.50 & 60 & 0.15 & 0.05 (HRS-A) \\
\hline & & & & 60 & 0.07 & 0.91 (LR1-A) \\
\hline${ }^{12} \mathrm{CO}$ & $J=3-2$ & $15^{\prime \prime}$ & 0.25 & 60 & 0.40 & 0.04 \\
\hline${ }^{13} \mathrm{CO}$ & $J=3-2$ & $15^{\prime \prime}$ & 0.28 & 180 & 0.30 & 0.04 \\
\hline
\end{tabular}

Note. $-{ }^{a}$ Main beam efficiency; ${ }^{b}$ Integration time per pointing; ${ }^{c}$ rms noise in antenna temperature; ${ }^{d}$ Channel separation, where prefix HR and LR refers to High and Low Resolution Spectrometers.

$J=1-0$ and $J=2-1$ lines, respectively) and a wide band lowresolution spectrometer (LRS) with 1440 channels, bandwidth $1000 \mathrm{MHz}, 700 \mathrm{kHz}$ channel separation (corresponding to $1.82 \mathrm{~km} \mathrm{~s}^{-1}$ and $0.91 \mathrm{~km} \mathrm{~s}^{-1}$ for the ${ }^{12} \mathrm{CO} J=1-0$ and $J=2$ 1 lines, respectively). The observed velocity intervals ranged from -45 to $+60 \mathrm{~km} \mathrm{~s}^{-1}$ and from -19 to $+35 \mathrm{~km} \mathrm{~s}^{-1}$ for the HRS $J=1-0$ and $J=2-1$ observations, respectively. The wide band observations covered the velocity intervals $(-1200$, $+1200) \mathrm{km} \mathrm{s}^{-1}(J=1-0)$ and $(-600,+600) \mathrm{km} \mathrm{s}^{-1}(J=2-1)$.

Two different samplings were used in the $J=1-0$ and $J=2-1$ line observations, one intended to have a broad view of the gas around G349.7+0.2, covering a $180^{\prime \prime} \times 180^{\prime \prime}$ area centered at $17^{\mathrm{h}} 18^{\mathrm{m}} 00^{\mathrm{s}},-37^{\circ} 26^{\prime} 25^{\prime \prime}(\mathrm{J} 2000)$, with a grid spacing of $45^{\prime \prime}$ and a second one, more detailed, consisting of 77 pointings covering an approximate area of $80^{\prime \prime} \times 120^{\prime \prime}$ around the same reference position with a grid sampling of $12^{\prime \prime}$ in both coordinates. The whole field was observed twice with an integration time of $30 \mathrm{~s}$ per position each time. The data were taken in a position switched mode, with an off position selected to be relatively free of emission, about half a degree away in right ascension and declination. The instrumental parameters are summarized in Table 1, where we list the antenna half-power beamwidth, main-beam efficiency of the telescope and spectral resolution at the observed line frequency, together with the attained noise level.

The observations in the ${ }^{12} \mathrm{CO} J=3-2(345.796 \mathrm{GHz})$ line were performed using the $345 \mathrm{GHz}$ SIS receiver with the high resolution (HRS) acousto optical spectrometer (2000 channels, bandwidth $80 \mathrm{MHz}$, channel separation $41.7 \mathrm{kHz}$, corresponding to $0.036 \mathrm{~km} \mathrm{~s}^{-1}$ at $\left.345 \mathrm{GHz}\right)$. The velocity interval covered at this frequency was $(-28,+45) \mathrm{km} \mathrm{s}^{-1}$. The ${ }^{12} \mathrm{CO} J=3-$ 2 line observations consist of 135 pointings that cover an area of $64^{\prime \prime} \times 120^{\prime \prime}$ with a grid spacing of $8^{\prime \prime}$. The integration time was 1 minute per pointing.

In addition, towards the center of the field, coinciding with the peak intensity of the different transitions, single spectra were obtained in ${ }^{13} \mathrm{CO} J=2-1(220.399 \mathrm{GHz})$ and ${ }^{13} \mathrm{CO} J=3-$ $2(330.588 \mathrm{GHz})$.
The pointing was checked once during each observing run using the AH Sco and VX Sgr SiO maser sources. The pointing errors were typically about $3^{\prime \prime}$. The system was calibrated at regular intervals yielding corrected antenna temperature $T_{\mathrm{A}}^{*}$. The data in this paper are given as main-beam brightness temperature $T_{\mathrm{mb}}$, where $T_{\mathrm{mb}}=T_{\mathrm{A}}^{*} / \eta$ and $\eta$ is the main beam efficiency at the corresponding frequency, as listed in Table 1.

\subsection{SNR G18.8+0.3}

The observations towards the SNR G18.8+0.3 were also carried out with the $15 \mathrm{~m}$ SEST Telescope in the ${ }^{12} \mathrm{CO} J=1-0$ and $J=2-1$ lines simultaneously. The high and low resolution acousto-optical spectrometers were used centered around $V_{\mathrm{LSR}}=20 \mathrm{~km} \mathrm{~s}^{-1}$. The observations were done in the positionswitching mode, covering a $10.5 \times 4.5$ area with a grid spacing of $45^{\prime \prime}$. A total of 105 positions was observed with an integration time of $15 \mathrm{~s}$ per pointing. The observational parameters are all similar to those listed for $\mathrm{G} 349.7+0.2$ for these ${ }^{12} \mathrm{CO}$ lines, except for the noise level, which turns out to be about $0.2 \mathrm{~K}$ and $0.3 \mathrm{~K}$ for the low and high resolution data respectively.

All spectra were processed using the XSpec software package developed at Onsala Space Observatory. Images were produced using the AIPS package. The spectra were Hanning smoothed to improve the signal-to-noise ratio.

\section{Results for SNR G349.7+0.2}

Figure 3 displays the spectra obtained with the high resolution spectrometer (HRS) towards the center of the surveyed area $\left(17^{\mathrm{h}} 18^{\mathrm{m}} 00^{\mathrm{s}},-37^{\circ} 26^{\prime} 25^{\prime \prime}, \mathrm{J} 2000\right)$ in all the observed lines. We find that the ${ }^{12} \mathrm{CO}$ lines (upper panels) are relatively narrow and quite symmetrical, while the ${ }^{13} \mathrm{CO}$ transitions (lower panels), mapping the optically thin material, show a red wing asymmetry. The main $\mathrm{CO}$ peaks can be fitted by Gaussians centered around $(16.2 \pm 0.2) \mathrm{km} \mathrm{s}^{-1}$, with average line widths at zero intensity of $8 \mathrm{~km} \mathrm{~s}^{-1}\left(\sim 4.5 \mathrm{~km} \mathrm{~s}^{-1} F W H M\right)$. A double-peaked feature is observed in the ${ }^{12} \mathrm{CO} J=1-0$ and 2-1 lines near $-10 \mathrm{~km} \mathrm{~s}^{-1}$, which can be fitted by two Gaussians 


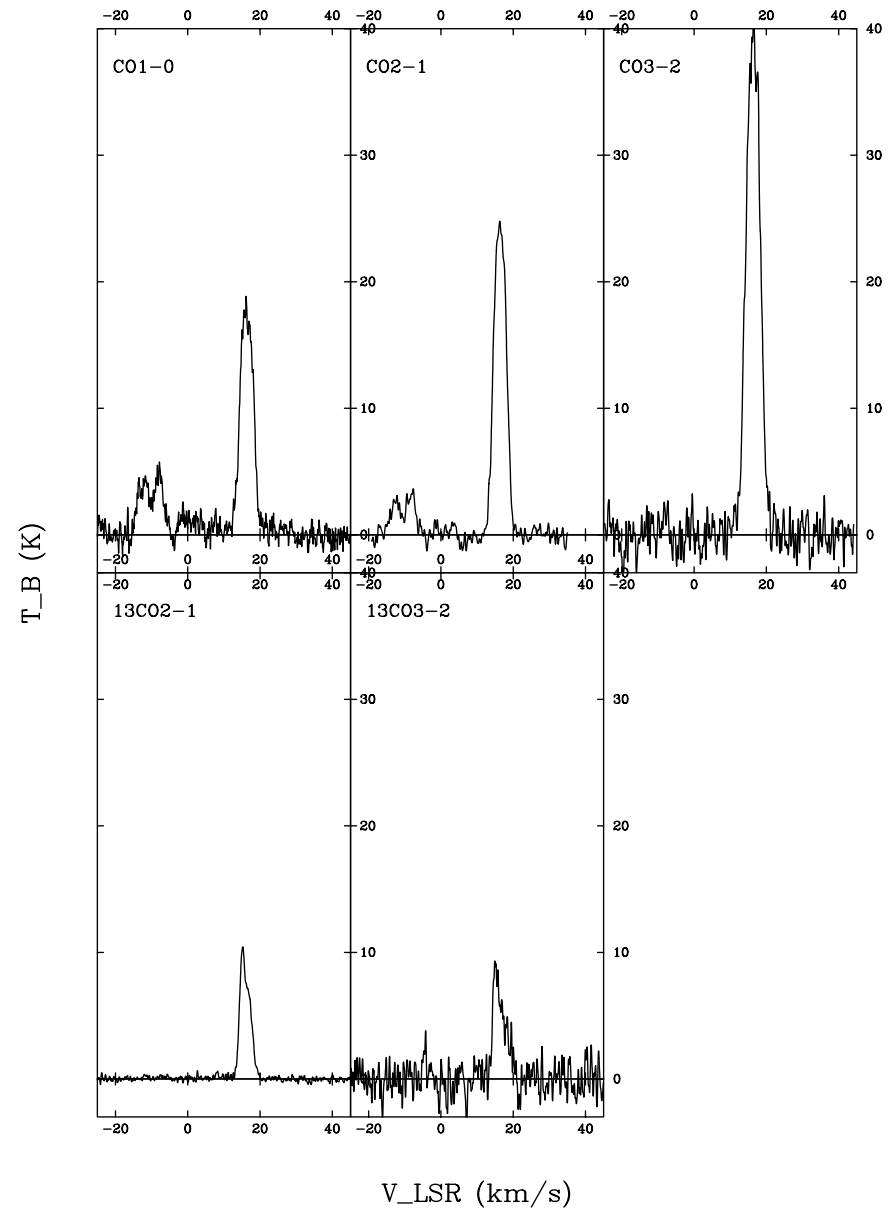

Fig. 3. Spectra of all the observed lines obtained towards the central position $17^{\mathrm{h}} 18^{\mathrm{m}} 00^{\mathrm{s}},-37^{\circ} 26^{\prime} 25^{\prime \prime}(\mathrm{J} 2000)$ in the SNR G349.7+0.2.

centered near $-8 \mathrm{~km} \mathrm{~s}^{-1} \quad\left(F W H M \sim 3 \quad \mathrm{~km} \mathrm{~s}^{-1}\right)$ and at $-12.5 \mathrm{~km} \mathrm{~s}^{-1}\left(F W H M \sim 3.5 \mathrm{~km} \mathrm{~s}^{-1}\right)$, respectively.

We have inspected the $180^{\prime \prime} \times 180^{\prime \prime}$ area observed in the ${ }^{12} \mathrm{CO} J=2-1$ line (with a $45^{\prime \prime}$ grid spacing) to investigate the global distribution of the molecular emission in this transition. A feature roughly circular in shape appears centered on the $\mathrm{SNR}$, in a similar way to the ${ }^{12} \mathrm{CO} J=1-0$ distribution shown by Reynoso \& Mangum (2000) and Lazendic et al. (2002). This cloud is part of a large molecular ring, as shown by Reynoso \& Mangum (2001).

We have also analyzed the broad-band low spectral resolution (LSR) data obtained in the ${ }^{12} \mathrm{CO} J=1-0$ and $J=2-1$ transitions (between $\pm 1200 \mathrm{~km} \mathrm{~s}^{-1}$ for $J=1-0$ and $\pm 600 \mathrm{~km} \mathrm{~s}^{-1}$ for $J=2-1$ ) looking for higher velocity features that could be associated with G349.7+0.2. Figure 4 shows a broad-band ${ }^{12} \mathrm{CO} J=1-0$ spectrum obtained from the average of all observed spectra towards G349.7+0.2. Additional velocity components are visible near $-110,-65$ and $-20 \mathrm{~km} \mathrm{~s}^{-1}$. The components at -65 and at $-20 \mathrm{~km} \mathrm{~s}^{-1}$ can be related to foreground Galactic gas emission (our line of sight to G349.7+0.2 crosses most of the Galaxy through the Galactic plane). The emission around $-110 \mathrm{~km} \mathrm{~s}^{-1}$ will be analyzed below in relation with $\mathrm{G} 349.7+0.2$.

The present high-angular resolution observations enable us to map in detail the molecular material towards the center

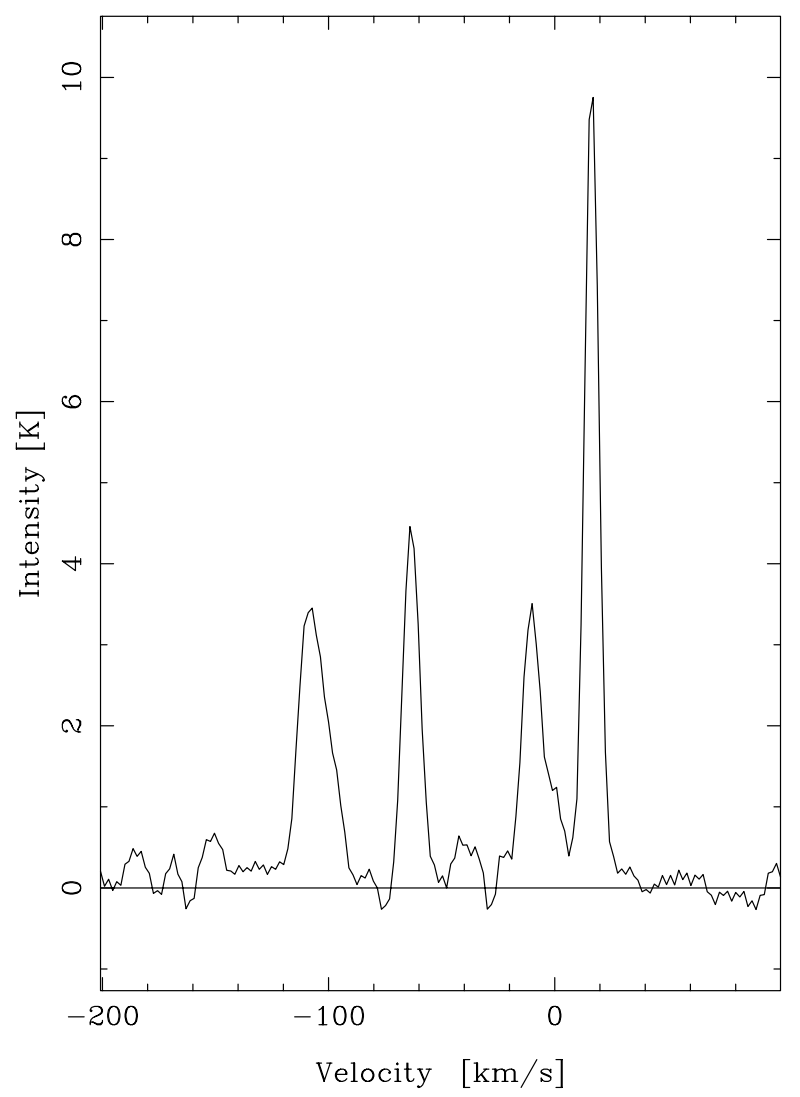

Fig. 4. ${ }^{12} \mathrm{CO} J=1-0$ average spectrum obtained towards $\mathrm{G} 349.7+0.2$. The peaks near -65 and $-10 \mathrm{~km} \mathrm{~s}^{-1}$ can be associated with emission from the Sagittarius and Norma galactic arms. The peak near $+16 \mathrm{~km} \mathrm{~s}^{-1}$ corresponds to the cloud at $22.4 \mathrm{kpc}$ associated with $\mathrm{G} 349.7+0.2$.

of G349.7+0.2. From the closely sampled observations (with $12^{\prime \prime}$ and $8^{\prime \prime}$ grid-spacing) we have produced ${ }^{12} \mathrm{CO} J=1-$ $0, J=2-1$ and $J=3-2$ line maps every $\sim 1 \mathrm{~km} \mathrm{~s}^{-1}$ between $\sim+12$ and $\sim+19 \mathrm{~km} \mathrm{~s}^{-1}$, the velocity interval where significant emission is observed in all three lines. The individual maps were obtained by integrating the brightness temperature over intervals of 9,18 and 27 channels for the $J=1-0, J=2-1$ and $J=3-2$ transitions, respectively. All three transitions peak near $+16 \mathrm{~km} \mathrm{~s}^{-1}$. In the ${ }^{12} \mathrm{CO} J=1-0$ transition, the cloud appears elongated with a single maximum around $17^{\mathrm{h}} 18^{\mathrm{m}} 00^{\mathrm{s}},-37^{\circ} 26^{\prime}$ $36^{\prime \prime}$ (Fig. 5). The ${ }^{12} \mathrm{CO} J=2-1$ cloud has two maxima (Fig. 6) centered near $17^{\mathrm{h}} 18^{\mathrm{m}} 00^{\mathrm{s}},-37^{\circ} 26^{\prime} 14^{\prime \prime}$ to the north and around $17^{\mathrm{h}} 18^{\mathrm{m}} 00^{\mathrm{s}},-37^{\circ} 27^{\prime} 00^{\prime \prime}$ to the south. The distribution of the ${ }^{12} \mathrm{CO} J=3-2$ emission (Fig. 7) also traces a cloud elongated in the $\mathrm{N}-\mathrm{S}$ direction, with the northern peak centered around $17^{\mathrm{h}} 18^{\mathrm{m}} 00^{\mathrm{s}},-37^{\circ} 26^{\prime} 20^{\prime \prime}$ and the southern feature less conspicuous than in the case of the $J=2-1$ line. A peculiar distortion in the shape of the $J=3-2$ cloud is evident in the maps at $v=+15.4,16.4$ and $17.4 \mathrm{~km} \mathrm{~s}^{-1}$.

Table 2 summarizes the observed and derived parameters for the cloud as observed in the three different rotational transitions. The central velocities and peak temperatures were obtained from Gaussian fittings to the spectra.

The associated molecular mass was estimated through three different methods: (a) making use of the ${ }^{13} \mathrm{CO} J=2-1$ and 


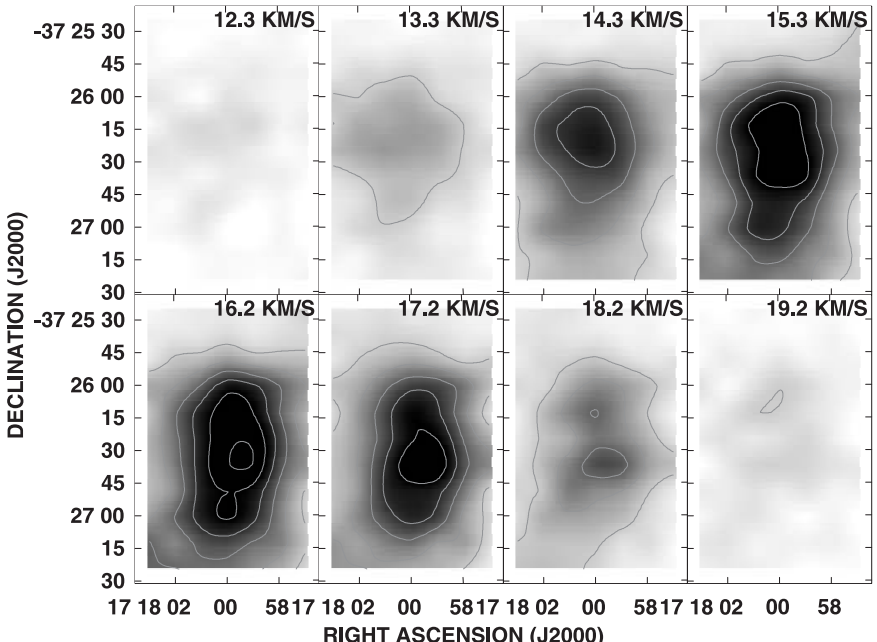

Fig. 5. ${ }^{12} \mathrm{CO} J=1-0$ emission towards the center of G349.7+0.2. The greyscale, representing the antenna temperature distribution, varies linearly between 0 and $10 \mathrm{~K}$. The plotted isocontours correspond to 2 , $4,6,8,10$ and $12 \mathrm{~K}$.

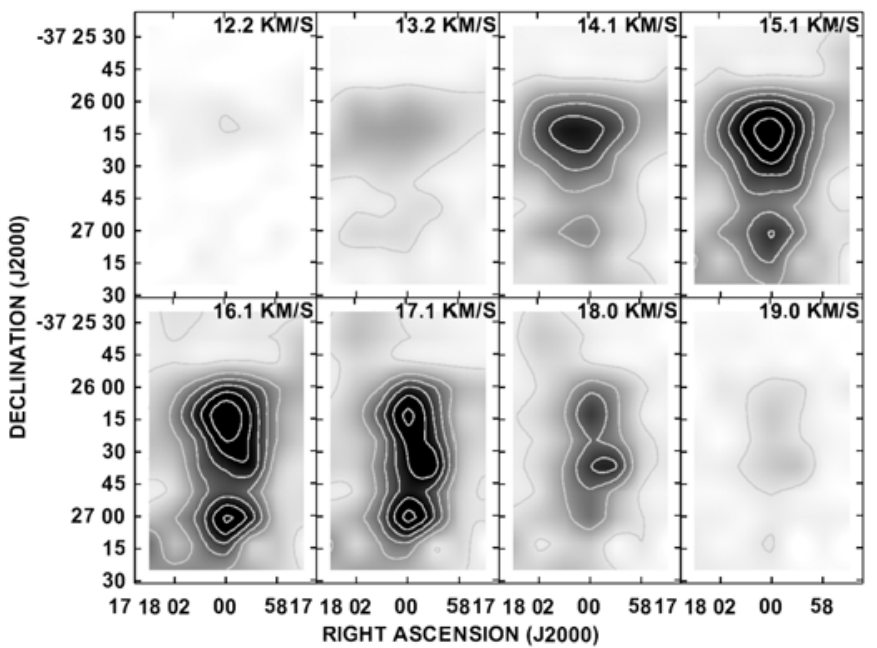

Fig. 6. ${ }^{12} \mathrm{CO} J=2-1$ emission towards the center of G349.7+0.2. The greyscale, representing the antenna temperature distribution, varies linearly between 0 and $10 \mathrm{~K}$. The plotted isocontours correspond to 1 , $2,4,6,8,10,12$ and $14 \mathrm{~K}$.

$J=3-2$ spectra obtained towards G349.7+0.2; (b) from the integrated brightness temperature of the ${ }^{12} \mathrm{CO} J=1-0$ line; and (c) through the virial theorem, by assuming that the cloud is in virial balance between turbulent velocity dispersion and selfgravity.

(a) For the calculations carried out using the observed ${ }^{13} \mathrm{CO}$ $J=2-1$ spectra, we have assumed that all molecules along the line of sight possess a uniform excitation temperature $T_{\mathrm{ex}}$ in the $J=2-1$ transition, and that different isotopic species have the same $T_{\text {ex }}$. The excitation temperature was calculated from the ${ }^{12} \mathrm{CO} J=2-1$ peak assuming that there is local thermodynamical equilibrium and that the line is optically thick, using the following relation:

$$
T_{\text {ex }}(\mathrm{K})=\frac{11.06}{\ln \left(\frac{1}{\frac{T_{\mathrm{B}}}{11.06}+0.0169}+1\right)}
$$

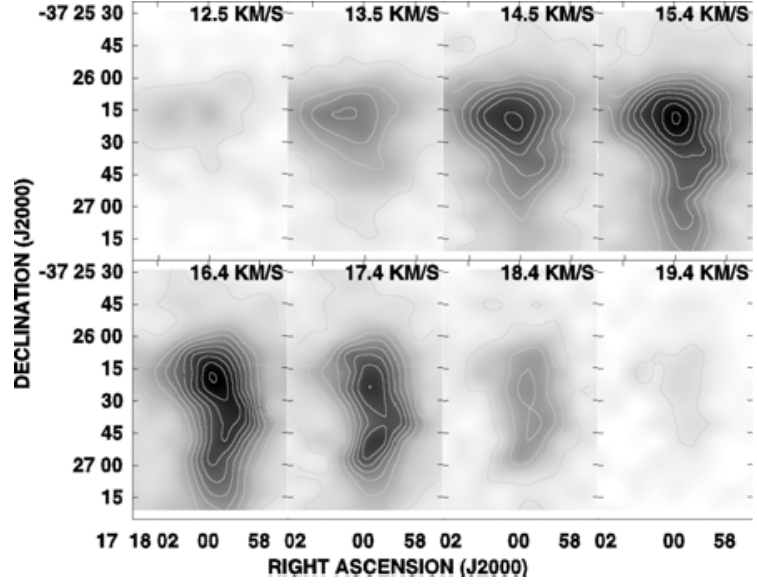

Fig. 7. ${ }^{12} \mathrm{CO} J=3-2$ emission towards the center of $\mathrm{G} 349.7+0.2$. The greyscale, representing the antenna temperature distribution, varies linearly between 0 and $10 \mathrm{~K}$. The plotted isocontours correspond to 1 , $2,3,4,5,6,7,8,9,10,11$ and $12 \mathrm{~K}$. Note the distortion in the shape between $\sim+15$ and $\sim 17 \mathrm{~km} \mathrm{~s}^{-1}$.

where $T_{\mathrm{B}}$ is the main beam brightness temperature of the ${ }^{12} \mathrm{CO}$ $J=2-1$ peak.

Applying this relation, the excitation temperature for the ${ }^{12} \mathrm{CO} J=2-1$ transition is estimated to be $T_{\mathrm{ex}}=35.4 \mathrm{~K}$, thus confirming that this transition is probing higher excitation temperatures than the $J=1-0$ line.

The optical depth $\tau\left({ }^{13} \mathrm{CO}_{2-1}\right)$ is estimated from:

$\tau^{13}=-\ln \left[1-\frac{T_{B}^{13}}{10.6}\left(\left(\mathrm{e}^{10.6 / T_{\mathrm{ex}}}-1\right)^{-1}-0.02\right)^{-1}\right]$

where $T_{\mathrm{B}}^{13}$ is the main beam brightness temperature of the ${ }^{13} \mathrm{CO} J=2-1$ line peak. The derived optical depth for the ${ }^{13} \mathrm{CO} J=2-1$ line is $\tau^{13}=0.4$.

Finally, the ${ }^{13} \mathrm{CO}$ column density, estimated from the relation:

$\mathrm{N}\left({ }^{13} \mathrm{CO}\right)=1.51 \times 10^{14} \frac{T_{\mathrm{ex}} \mathrm{e}^{5.3 / T_{\text {ex }} \int \tau^{13}(v) \mathrm{d} v}}{1-\mathrm{e}^{-10.6 / T_{\text {ex }}}}$,

yields $3.8 \times 10^{16} \mathrm{~cm}^{-2}$. Using the fractional abundance $N\left(\mathrm{H}_{2}\right) / N\left({ }^{13} \mathrm{CO}\right)=5 \times 10^{5}$ (Dickman 1978), the $\mathrm{H}_{2}$ column density is estimated to be $N\left(\mathrm{H}_{2}\right)=1.9 \times 10^{22} \mathrm{~cm}^{-2}$. A total amount of molecular gas of $1.1 \times 10^{4} M_{\odot}$ is estimated. In the mass calculation we have used a mean molecular weight per $\mathrm{H}_{2}$ molecule of 2.72 times the mass of the $\mathrm{H}$ atom, which takes into account helium and heavy elements content in the cloud, and we have adopted a distance to G349.7+0.2 of $22.4 \mathrm{kpc}$.

(b) The second way to estimate the molecular mass content of the cloud is from the integration of the brightness temperature of ${ }^{12} \mathrm{CO} J=1-0$ between 12 and $20 \mathrm{~km} \mathrm{~s}^{-1}$, and by applying the relations

$$
\begin{aligned}
& W(\mathrm{CO})=\int T_{\mathrm{B}} \mathrm{d} v \\
& M\left(M_{\odot}\right)=5.65 \times 10^{-21} X W_{\mathrm{CO}} \theta^{2} D^{2}
\end{aligned}
$$

with the distance $D$ in $\mathrm{kpc}$, the angular radius $\theta$ in $\operatorname{arcmin}$ and adopting a molecular mass calibrating ratio $X=N\left(\mathrm{H}_{2}\right) / W(\mathrm{CO})$ 
Table 2. Observed and derived parameters for the ${ }^{12} \mathrm{CO}$ clouds towards G349.7+0.2.

\begin{tabular}{|c|c|c|c|c|c|}
\hline & ${ }^{12} \mathrm{CO} \mathrm{J}=1-0$ & ${ }^{12} \mathrm{CO} J=2-1$ & ${ }^{12} \mathrm{CO} J=3-2$ & ${ }^{13} \mathrm{CO} J=2-1$ & ${ }^{13} \mathrm{CO} \mathrm{J}=3-2$ \\
\hline$T_{\text {peak }}^{B}(\mathrm{~K})$ & 18.2 & 30 & 44 & 10 & 10 \\
\hline$V_{\text {cent }}\left(\mathrm{km} \mathrm{s}^{-1}\right)$ & 16.2 & 16.3 & 16.3 & 15.6 & 15.5 \\
\hline$\Delta \mathrm{v}\left(\mathrm{km} \mathrm{s}^{-1}\right)$ & 4.2 & 3.9 & 4.4 & 3.4 & 4 \\
\hline Angular diameter (') & 0.58 & 0.55 & 0.51 & & \\
\hline Linear diameter $(\mathrm{pc})$ & 7.6 & 7.2 & 6.7 & & \\
\hline$W(\mathrm{CO})\left(\mathrm{K} \mathrm{km} \mathrm{s}^{-1}\right)$ & 50.9 & 69.8 & 110.0 & & \\
\hline$T_{\mathrm{ex}}(\mathrm{K})$ & 22 & 35 & 52 & & \\
\hline Molecular mass $\left(M_{\odot}\right)$ & $1 \times 10^{4}$ & & & & \\
\hline Volume density $\left(\mathrm{cm}^{-3}\right)$ & $1 \times 10^{3}$ & & & & \\
\hline Total column density $\left(\mathrm{cm}^{-2}\right)$ & $6.9 \times 10^{22}$ & & & & \\
\hline$R_{(2-1) /(1-0)}$ & $\sim 1.5$ & & & & \\
\hline$R_{(3-2) /(2-1)}$ & $\sim 2.4$ & & & & \\
\hline$R_{(3-2) /(1-0)}$ & $\sim 3.5$ & & & & \\
\hline
\end{tabular}

equal to $(2.7 \pm 0.9) \times 10^{20}$ molecules $\mathrm{cm}^{-2} \mathrm{~K}^{-1} \mathrm{~km}^{-1} \mathrm{~s}$, a value appropriate for the location of $\mathrm{G} 349.7+0.2$ in the outer Galaxy (galactocentric radius of $14 \mathrm{kpc}$ ) (Bloemen et al. 1986; Digel et al. 1996). From these relations we obtained $M=9.7 \times$ $10^{3} M_{\odot}$.

(c) Finally, by applying the virial theorem

$M_{\mathrm{tot}} \simeq 2 \frac{R_{\mathrm{tot}} v^{2}}{G}$

and using an average radius of $3.6 \mathrm{pc}$ and a mean velocity dispersion of $4 \mathrm{~km} \mathrm{~s}^{-1}$ for the molecular cloud, we derive $M=2.3 \times 10^{4} M_{\odot}$. This estimate agrees within a factor of 2 with the former mass calculations, suggesting that the cloud may be close to the virial equilibrium.

We can therefore conclude that in this case all three methods produce very similar values for the molecular mass. The errors involved in the mass calculation are high, of the order of $50 \%$, with the largest sources of uncertainties being the distance (about 10\%), and possible variations (up to $30 \%$ ) in the adopted value for the calibrating ratio $\mathrm{X}$ (in method (b)).

The luminosity is $L_{\mathrm{CO}} \sim 2.4 \times 10^{3} \mathrm{~K} \mathrm{~km} \mathrm{~s}^{-1} \mathrm{pc}^{2}$. The volume density is $n \sim 10^{3}$ molecules $\mathrm{cm}^{-3}$, in agreement with the value obtained by Reynoso \& Mangum (2000) based on ${ }^{12} \mathrm{CO} J=1-0$ observations. The derived molecular density suggests that we are mainly detecting ambient pre-shock gas, as observed and modelled by Lazendic et al. (2002), instead of the highly compressed post-shock gas. This is compatible with the relatively narrow width of the lines observed. For the postshock gas a density of $n \sim 10^{5}-10^{6} \mathrm{~cm}^{-3}$ is proposed by these authors.

The ratio between the integrated intensities at different transitions is a useful parameter to investigate variations of physical conditions within a cloud and to trace shocked gas regardless of the gas amount (Seta et al. 1998). The higher transitions of ${ }^{12} \mathrm{CO}$ are valuable probes of the denser and warmer material of the interacting cloud. After convolving the ${ }^{12} \mathrm{CO}$ $J=2-1$ and the ${ }^{12} \mathrm{CO} J=3-2$ data to the same angular resolution as the $J=1-0$ data, we find that at an angular resolution of $45^{\prime \prime}$ the integrated line ratios (listed in Table 2) are almost uniform across the observed region. The $R_{(2-1) /(1-0)}$ values are comparable to the ratios observed in shocked clouds associated with the SNRs W44 (1.3), IC 443 (1.3-4), and HB 21 (1.6-2.3) (Seta et al. 1998; Koo et al. 2001). The high line ratios observed in G349.7+0.2 can be an indicator of shocked gas, in spite of the fact that the spectra are not broadened.

\subsection{Comparison with emission at other wavelengths}

Figures $8 \mathrm{a}$ and $8 \mathrm{~b}$ display the distribution of the $J=2-1$ and $J=3-2$, respectively, as obtained from the integration over the interval $(12,19) \mathrm{km} \mathrm{s}^{-1}$, overlapping the radio continuum emission of G349.7+0.2 at $20 \mathrm{~cm}$ (from Brogan et al. 2000). In Fig. 8c the ${ }^{12} \mathrm{CO} J=3-2$ integrated intensity is compared with the X-ray emission from the ASCA-SIS observations between $0.5-10 \mathrm{keV}$ (in greys) (from Slane et al. 2002) and the radio continuum (in light grey contours). The outermost contour of the molecular emission plotted is the last closed contour.

Different aspects can be noticed from the present correlations:

1) Comparison with radio continuum emission: the morphology of the molecular cloud near $v_{\mathrm{LSR}} \simeq+16 \mathrm{~km} \mathrm{~s}^{-1}$ shows good correlation with some prominent radio continuum features. The bright synchrotron ridge that crosses G349.7+0.2 from NE to SW mimics the shape of the eastern half of the cloud, mainly as it is traced in the $J=3-2$ line from $v \sim$ $+15 \mathrm{~km} \mathrm{~s}^{-1}$ to $v \sim+17 \mathrm{~km} \mathrm{~s}^{-1}$ (see Figs. 7 and 8b). This morphological agreement suggests that we are observing the mutual signatures of a strong SN shock/molecular cloud interaction. On the one side, the SN shock running into the molecular cloud with an approximate east-west direction as apparent in the plane of the sky, has compressed and distorted the interstellar cloud. Conversely, the encounter of the SN shock with denser gas may have enhanced the brightness of the radio filament either by the amplification of the magnetic field (because of higher compression of the magnetic lines) or because of the stimulated particle acceleration taking place at the shock front, or both mechanisms simultaneously. Concerning the brightest radio continuum feature in the south (near $17^{\mathrm{h}} 18^{\mathrm{m}} 01^{\mathrm{s}},-37^{\circ}$ $27^{\prime} 00^{\prime \prime}$, close to the X-ray maximum as shown in Fig. 8c), it appears near to, but not coincident with, the southern peak of 


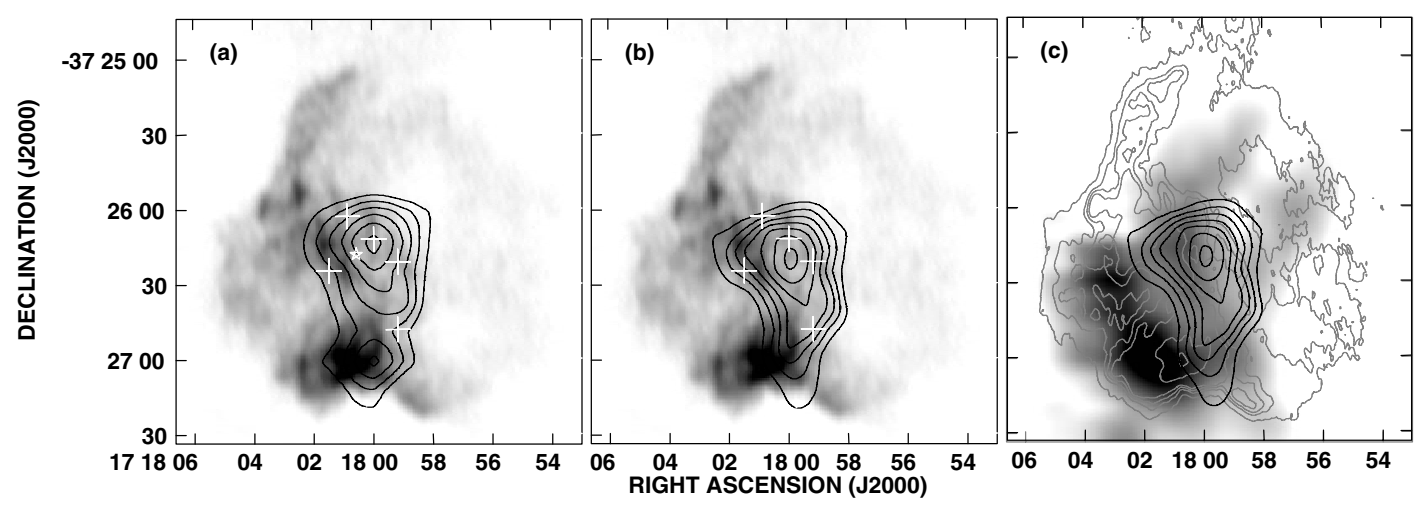

Fig. 8. a) distribution of ${ }^{12} \mathrm{CO} J=2-1$ towards $\mathrm{G} 349.7+0.2$ as obtained from the integration between 12 and $19 \mathrm{~km} \mathrm{~s}^{-1}$ in grey contours overlapping the radio continuum emission displayed in greyscale (from Brogan et al. 2000); the white crosses indicate the locations of the $\mathrm{OH}$ $(1720 \mathrm{MHz})$ masers. The white star shows the location of the infrared point source IRAS J17146-3723. The contour lines are traced at 40, $55,70,85,100$ and $115 \mathrm{~K} \mathrm{~km} \mathrm{~s}^{-1}$; b) the same for the ${ }^{12} \mathrm{CO} J=3-2$ transition. The contour lines are traced at $60,80,100,120,140,160$ and $180 \mathrm{~K} \mathrm{~km} \mathrm{~s}^{-1}$; c) X-ray emission from the ASCA-SIS observations between $0.5-10 \mathrm{keV}$ is shown in greyscale (from Slane et al. 2002) with the radio continuum emission in light grey contours (plotted contours are 2, 10, 15, 20, 40, 60, 80 and $100 \mathrm{mJy} / \mathrm{beam}$ ) and the ${ }^{12} \mathrm{CO} J=3-2 \mathrm{cloud}$ in black contours.

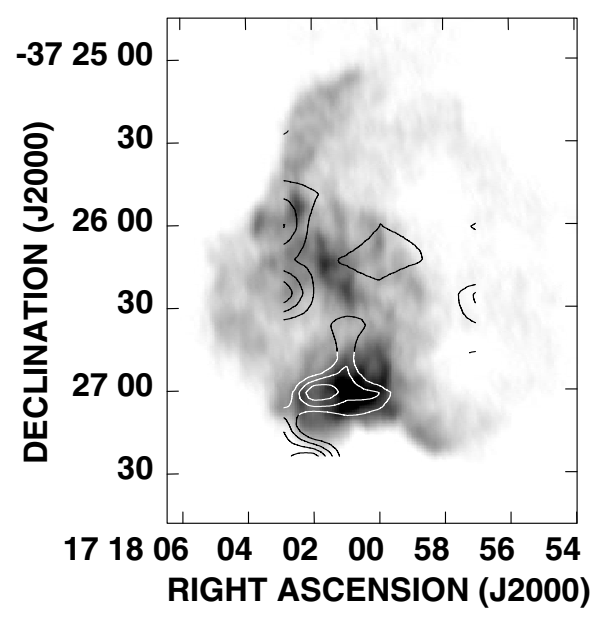

Fig. 9. Overlay of radio continuum emission of G349.7+0.2 with the ${ }^{12} \mathrm{CO} J=1-0$ emission integrated over 5 channels around $v_{\mathrm{LSR}}=$ $-109 \mathrm{~km} \mathrm{~s}^{-1}$. The plotted contours correspond to $16,17,18,19$ and $20 \mathrm{~K} \mathrm{~km} \mathrm{~s}^{-1}$.

the ${ }^{12} \mathrm{CO} J=2-1$ cloud (Fig. 8a). In what follows we discuss a possible origin for this feature.

2) In an inspection of the low spectral resolution observations in the $J=1-0$ and $J=2-1$ transitions, a high-velocity cloud was discovered in the $J=1-0$ line, centered at $v=$ $-109 \mathrm{~km} \mathrm{~s}^{-1}$, which overlaps the radio maximum (Fig. 9). In $J=2-1$ some traces of the feature are also detected. Note that besides the analyzed cloud near $v \sim+16 \mathrm{~km} \mathrm{~s}^{-1}$ this is the only feature detected in ${ }^{12} \mathrm{CO}$ within the whole observed velocity range (up to $\pm 1200 \mathrm{~km} \mathrm{~s}^{-1}$ ) that has any positional correspondence with the southern radio and X-ray maxima. The characteristic parameters of this high-velocity cloud are $M \sim 1.5 \times 10^{3} M_{\odot}$ and $n \sim 1.5 \times 10^{3} \mathrm{~cm}^{-3}$, if we assume that the cloud is effectively associated with G349.7+0.2 and therefore located as far away as $22 \mathrm{kpc}$. To test if the observed emission comes from shocked accelerated gas, we calculated the line ratio $R_{(2-1) /(1-0)}$, which turns out to be $\sim 0.5$. As discussed before, higher ratios are expected for shocked gas. In addition, the association of this molecular feature with the SNR is difficult to prove because this is the velocity range corresponding to the tangent point for $l \sim 349^{\circ}$ so velocity crowding is important. In what follows we restrict our analysis to the molecular cloud detected near $v \sim+16 \mathrm{~km} \mathrm{~s}^{-1}$.

3) Comparison with infrared emission: we have calculated the $60 \mu \mathrm{m} / 100 \mu \mathrm{m}$ IR ratio from the IRAS Sky Survey Atlas high resolution (HIRES) images obtained with 20 iterations of the algorithm (Cao et al. 1997). An enhancement in the 60/100 IR color is observed near the center of the $\mathrm{CO}$ cloud which is compatible with shock-heated dust. A radiative SN shock can be responsible for significant grain destruction, and different mechanisms, such as nonthermal sputtering and grain-grain collisions, preferentially destroy large grains rather than small ones (Seab \& Shull 1983). From the IR color ratio estimated from the HIRES images we have calculated the dust temperature towards the center of the SNR to be $T_{\text {dust }}=37 \mathrm{~K}$. This value is similar to the temperature of shock-heated dust found in other SNRs (Arendt et al. 1992; Castelletti et al. 2003). We have also estimated the dust content in the cloud (assuming a grain mass of $4 \times 10^{-14} \mathrm{~g}$ ) $M_{\text {dust }} \simeq 110 M_{\odot}$. The dust-to-gas ratio is then 0.011 , comparable to the normal interstellar medium value.

The point infrared source IRAS 17146-3723 $\left(17^{\mathrm{h}} 18^{\mathrm{m}} 0.5626,-37^{\circ} 26^{\prime} 17^{\mathrm{s}} .97 \mathrm{~J} 2000\right)$ (shown by a white star in Fig. 8a), has spectral characteristics compatible with a star forming region or with shock-heated dust according to Junkes et al.'s (1992) criteria. It is located very close to the position where the radio filament is brighter and the $J=3-2$ cloud looks distorted, giving further support to the hypothesis of a physical interaction between the SN shock and the dense molecular cloud.

4) Comparison with X-ray emission: the X-ray emission between 0.5 and $10 \mathrm{keV}$ (from Slane et al. (2002)) fills most of the interior of G349.7+0.2 and peaks near the SE corner of the SNR, coinciding with the southern radio maximum. 


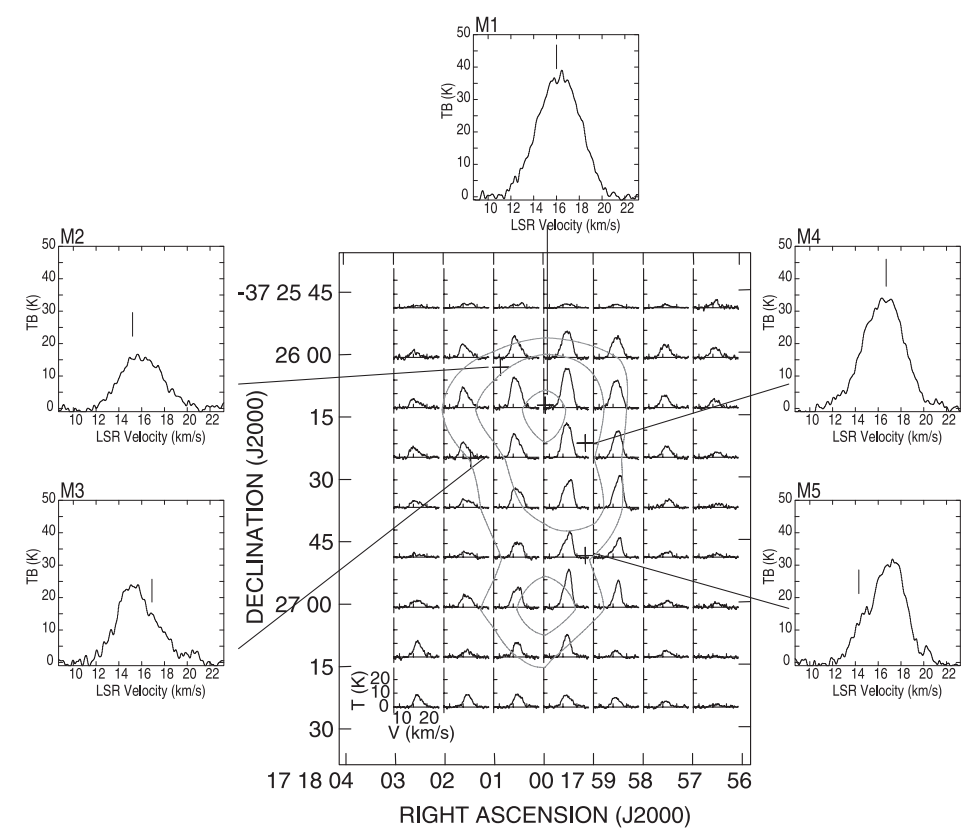

Fig. 10. ${ }^{12} \mathrm{CO} J=2-1$ spectra towards the center of $\mathrm{G} 349.7+0.2$, with a few isocontours showing the position of the CO cloud. The crosses show the locations of the $\mathrm{OH}(1720 \mathrm{MHz})$ masers. The five spectra that surround the central plot correspond to the ${ }^{12} \mathrm{CO} J=3-2$ emission averaged over a $1^{\prime}$ region around each maser.

No particular correspondence is observed between the molecular distribution and the X-ray emission. The diffuse central X-ray emission observed is consistent with a "mixedmorphology" nature for G349.7+0.2. Such SNRs are characterized by central thermal X-ray emission, surrounded by shelllike radio emission. It is known that most of these remnants are interacting with molecular clouds and have strong infrared line emission and OH (1720 MHz) masers (Rho \& Petre 1998; Yusef-Zadeh et al. 2003). In general, it is expected that higher density regions in the interstellar medium correlate with minima in the X-rays because of the increased absorption. In the next section we discuss the implications of the lack of correlation observed in this case.

\subsection{Column density towards G349.7+0.2}

We used the low-spectral resolution (LSR) observations of the ${ }^{12} \mathrm{CO} J=1-0$ to investigate the absorbing material towards $\mathrm{G} 349.7+0.2$. From the integration of the $\mathrm{CO}$ gas between $-150 \mathrm{~km} \mathrm{~s}^{-1}$ and $+20 \mathrm{~km} \mathrm{~s}^{-1}$, the velocity interval that takes into account all the intervening gas between us and the extreme velocity where the associated $\mathrm{CO}$ cloud is detected, we obtain $N\left(\mathrm{H}_{2}\right) \simeq 5 \times 10^{22}$ molecules $\mathrm{cm}^{-2}$ (with variations across the observed field of the order of $\left.\pm 1 \times 10^{22} \mathrm{~mol} . \mathrm{cm}^{-2}\right)$. In this calculation a calibrating ratio $X=N\left(\mathrm{H}_{2}\right) / W(\mathrm{CO})=(2.7 \pm$ $0.9) \times 10^{20}$ molecules $\mathrm{cm}^{-2} \mathrm{~K}^{-1} \mathrm{ks}^{-1} \mathrm{~s}$ is adopted. Therefore the line-of-sight hydrogen column density, $\left.N=\left[2 N\left(\mathrm{H}_{2}\right)+N_{\mathrm{H}}\right)\right] \simeq$ $1.1 \times 10^{23} \mathrm{~cm}^{-2}$, where $N_{\mathrm{H}}$ in this direction of the Galaxy was estimated from the Bell Labs HI survey database (Stark et al. 1992). Yamauchi et al. (1998) and Slane et al. (2002) modelled the X-ray emission of $\mathrm{G} 349.7+0.2$ by a thin thermal emitting plasma with an absorbing column of $N \sim 6 \times 10^{22} \mathrm{~cm}^{-2}$. The radio and $\mathrm{X}$-rays results can be reconciled if we assume that the molecular cloud observed around $v=+16 \mathrm{~km} \mathrm{~s}^{-1}$ is located behind G349.7+0.2 (in the line-of-sight direction). In effect, if we subtract the contribution of the associated $\mathrm{CO}$ cloud calculated in the previous section to be $N\left(\mathrm{H}_{2}\right)=1.9 \times 10^{22} \mathrm{~cm}^{-2}$, then $N_{\text {tot }} \sim 6.9 \times 10^{22} \mathrm{~cm}^{-2}$, in better agreement with the constraints from the X-rays. We thus conclude that the SNR/molecular cloud interaction is taking place on the back side of the SNR. This model provides a natural explanation for the lack of absorption in the X-ray radiation toward the center of the SNR. Evaporation of part of this cloud in the interior of G349.7+0.2 would be responsible for the central X-ray emission, as proposed by Rho \& Petre (1998) for composite SNRs.

\subsection{Kinematical characteristics of the molecular cloud}

Figure 10 displays the set of ${ }^{12} \mathrm{CO} J=2-1$ spectra obtained across the $\left(80^{\prime \prime} \times 120^{\prime \prime}\right)$ observed field towards the center of G349.7+0.2. In this plot the bottom left corner of each spectrum is positioned at the respective RA-Dec.

Clear asymmetries can be observed when one moves from the center of the cloud to the borders. It can be appreciated that spectra of pointings located in the eastern half have preferentially a red wing broadening, while spectra to the west and south of the central line, exhibit a mirroring blue wing broadening. Similar behavior can be found in the ${ }^{12} \mathrm{CO} J=3-2$ spectra. This property is an indication of kinematical effects in the molecular cloud.

In Fig. 10 we have also plotted the ${ }^{12} \mathrm{CO} J=3-2$ spectra obtained towards the five $\mathrm{OH}(1720 \mathrm{MHz})$ masers (averaged over a $1^{\prime}$ region around every maser). The bar in every spectrum indicates the LSR peak velocity of the $\mathrm{OH}$ masers as derived from VLA $1720 \mathrm{MHz}$ observations carried out with a velocity resolution of $1.1 \mathrm{~km} \mathrm{~s}^{-1}$ (Frail et al. 1996). It is readily 
apparent that the maser peak velocity agrees very well with the local CO peak velocity within the quoted errors. In the cases of M 3 and M 5, two masers that coincide with the most asymmetric $\mathrm{CO}$ profiles, the maser peak velocity agrees with the radial velocity of a secondary, fainter component of the CO spectrum, which appears to be blended with the main one.

Our observations have revealed for the first time the internal structure of the molecular cloud associated with G349.7+0.2, and although the limited angular resolution does not make it possible to detect the post-shock gas (probably with broader wings), the consequences of the impact of the shock of this SNR on the neighbouring cloud are kinematically evident.

Several studies suggest that $\mathrm{OH}(1720 \mathrm{MHz})$ masers are more efficiently pumped in shocks that propagate transverse to the line of sight (Lockett et al. 1999). Wardle (1999) concludes that when the angle between the normal of a non-dissociative C-type shock and the line of sight is $\geq 80^{\circ}, \mathrm{N}_{\mathrm{OH}}$ is sufficient to permit the formation of $\mathrm{OH}(1720 \mathrm{MHz})$ masers.

Based on the present results, the following model can be proposed: the expanding shock struck a cloud placed on the back side, forming a large angle with the line of sight, and initiated the disruption of the cloud. The borders are the first regions affected by the process because they are less dense, and while the eastern portion is apparently being pushed in a direction away from us, the western side has internal motions mostly directed towards us.

In addition, when we compare the location of every maser with the CO distribution obtained exactly at the maser peak velocity, the masers always appear located in the periphery of the cloud, similar to what is observed in the SNRs W28 and W44 (Frail \& Mitchell 1998; Arikawa et al. 1999). In the case of SNR W44, the shock is clearly transverse to the line of sight. In the case of W28, however, there are masers associated not only with transverse shocks, but also with a shock impinging on a cloud located along the line of sight (Arikawa et al. 1999). Once the SN shock has overtaken a molecular cloud and penetrated it, the topology of the SN shock must be severely affected. Therefore although the shock front does not look perpendicular to the line of sight, as seems to be the case in G349.7+0.2, locally it may well have the right angle to excite maser emission.

\section{Results for SNR G18.8+0.3}

In this SNR, after a careful inspection of the CO database over the entire velocity range, we find that the only channels showing morphological and kinematical possible signatures of interaction between the supernova remnant and the surroundings are limited to the range $\sim+16.5$ to $+25 \mathrm{~km} \mathrm{~s}^{-1}$.

In Fig. 11 we display the distribution of the ${ }^{12} \mathrm{CO} J=2-$ 1 line emission in the mentioned velocity interval. Each image was obtained by averaging eight spectral channels (spanning $\simeq 0.6 \mathrm{~km} \mathrm{~s}^{-1}$ ). The velocity shown in the upper right corner of each image corresponds to the velocity of the first integrated channel. A few representative radio continuum contours are superimposed.

The improved angular resolution and signal-to-noise ratio of the new CO maps, as compared to the survey in
Dubner et al. (1999), reveals that the eastern cloud previously identified as cloud 3 is resolved into two components, one near $18^{\mathrm{h}} 24^{\mathrm{m}} 13^{\mathrm{s}},-12^{\circ} 27^{\prime} 50^{\prime \prime}(\mathrm{J} 2000.0)$ (hereafter "cloud A"), and the other near $18^{\mathrm{h}} 24^{\mathrm{m}} 10^{\mathrm{s}},-12^{\circ} 29^{\prime} 30^{\prime \prime}(\mathrm{J} 2000.0)$ (hereafter "cloud B").

In particular, cloud A shows an excellent correspondence with the brightest part of the continuum shell of G18.8+0.3. In order to remark this feature, in Fig. 12 we display the $\mathrm{CO}$ emission integrated between +17.6 and $+20.9 \mathrm{~km} \mathrm{~s}^{-1}$ in contour lines, while the radio continuum is displayed in greyscale. The perfect fitting observed between the outermost contour of cloud A and the radio continuum maximum allows us to suggest that they are in physical contact.

A Gaussian fit to the velocity profiles of the two $\mathrm{CO}$ clouds $\mathrm{A}$ and $\mathrm{B}$ in the $J=1-0$ transition yields peak temperatures of $15.3 \mathrm{~K}$ at $19.9 \mathrm{~km} \mathrm{~s}^{-1}$ for cloud $\mathrm{A}$, and $12.4 \mathrm{~K}$ at $19.7 \mathrm{~km} \mathrm{~s}^{-1}$ for cloud $\mathrm{B}$, in close agreement with the result of Dubner et al. (1999) $\left(19 \pm 1 \mathrm{~km} \mathrm{~s}^{-1}\right)$.

Applying the flat Galactic rotation curve of Fich et al. (1989; with $R_{\odot}=8.5 \mathrm{kpc}$ and $V_{\odot}=220 \mathrm{~km} \mathrm{~s}^{-1}$ ), with the adopted systemic velocity of $\sim 20 \mathrm{~km} \mathrm{~s}^{-1}$, we obtain kinematic distances of either $\sim 1.9$ or $\sim 14 \mathrm{kpc}$. The two possible distance values result from the distance ambiguity in the inner part of the Galaxy. The HI absorption profiles presented by Caswell et al. (1975) towards G18.8+0.3 indicate that this remnant is placed beyond the tangential point, thus in what follows we will adopt the farther distance of $14 \mathrm{kpc}$ even though the shortest distance had been used in our previous study (Dubner et al. 1999). With the revised distance to G18.8+0.3, the dynamical age of this SNR turns out to be almost $10^{5}$ yrs.

Table 3 summarizes the observed and derived parameters for the clouds. To obtain column densities and masses, we adopted the opacity $\tau\left({ }^{13} \mathrm{CO}\right)=0.46$ estimated in Dubner et al. (1999), and derived $T_{\text {ex }}$ from the current CO $J=1-0$ data assuming that there is local thermodynamic equilibrium and that the line is optically thick. The column density $N\left({ }^{13} \mathrm{CO}\right)$ is then estimated from the following relation (for the $J=1-0$ transition):

$N\left({ }^{13} \mathrm{CO}\right)=2.6 \times 10^{14} \frac{T_{\mathrm{ex}} \int \tau^{13}(v) \mathrm{d} v}{1-\mathrm{e}^{-5.3 / T_{\text {ex }}}} \mathrm{cm}^{-2}$.

The column density $N_{\mathrm{H}_{2}}$ of the clouds is then directly derived from the fractional abundance $N\left(\mathrm{H}_{2}\right) / N\left({ }^{13} \mathrm{CO}\right)=5 \times 10^{5}$ (Dickman 1978). Volume densities were estimated assuming spherical geometry.

Over the entire region, line velocity widths are of the order of $7 \mathrm{~km} \mathrm{~s}^{-1}$, characteristic of unperturbed gas. Even though there appear to be no traces of shocked molecular gas in the observed lines, the remarkable morphological agreement between the radio shell and the molecular cloud A is very suggestive of an actual physical association. Therefore, we have looked for additional indications of interaction through the $\mathrm{CO}$ $J=2-1 / 1-0$ intensity ratio $\left(R_{(2-1) /(1-0)}\right)$. We have divided the CO $J=2-1$ intensity integrated over a $2 \mathrm{~km} \mathrm{~s}^{-1}$ velocity interval around the systemic velocity of the cloud by the $\mathrm{CO}$ $J=1-0$ intensity integrated over the same velocity interval. The ratio map is shown in Fig. 13 in comparison with the radio continuum limb (grey contours) and the ${ }^{12} \mathrm{CO} J=1-0$ line 


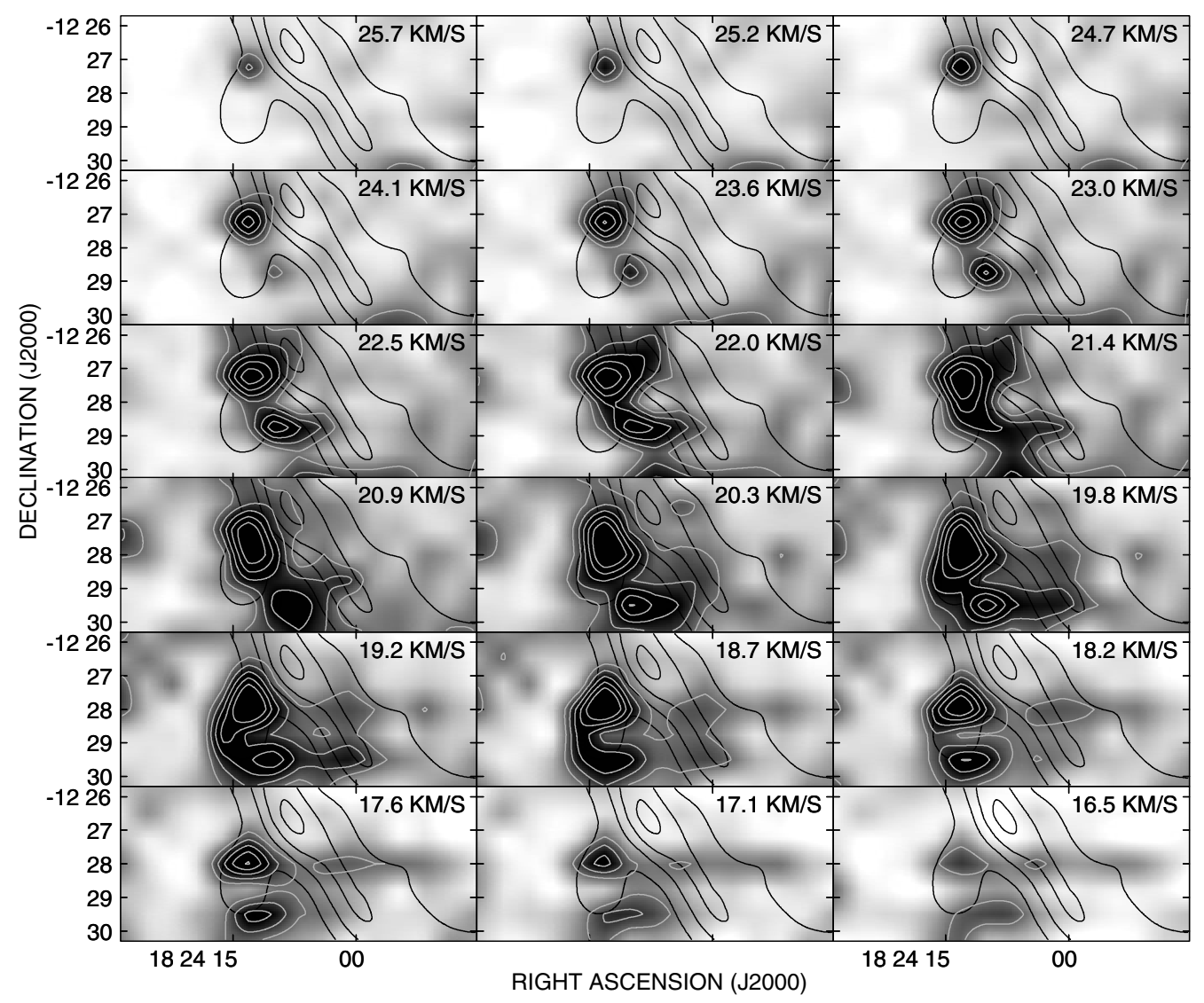

Fig. 11. ${ }^{12} \mathrm{CO} J=2-1$ emission distribution around the eastern protrusion in $\mathrm{G} 18.8+0.3$ (greys). The greyscale varies between 3 and $50 \mathrm{~K}$ in brightness temperature. Grey contours, traced at 30, 40,50, 60 and $70 \mathrm{~K}$, are included to better display the morphology of the associated features. Overlapping black contours representing the radio continuum emission are traced at $0.1,0.3,0.5 \mathrm{and} 0.7 \mathrm{Jy} / \mathrm{beam}$.

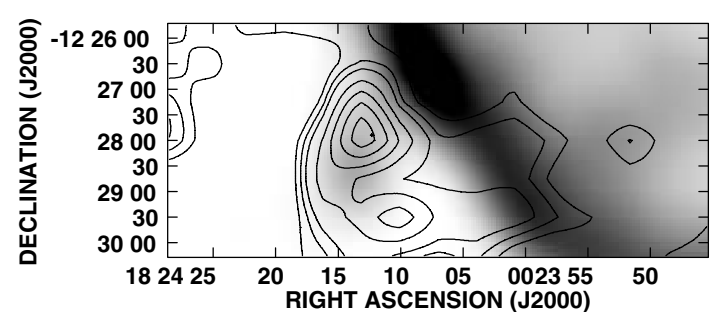

Fig. 12. Radio continuum emission towards $\mathrm{G} 18.8+0.3$ at $1.4 \mathrm{GHz}$ in greyscale with overlapping contours displaying the ${ }^{12} \mathrm{CO} J=2-1$ emission integrated over the velocity range $(+17.6,+20.9) \mathrm{km}^{-1}$. The plotted contours are $10,12.5,15,20,25,30,35$ and $40 \mathrm{~K} \mathrm{~km}^{-1}$.

distribution (black contours). Values of $R_{(2-1) /(1-0)}$ vary from 0.8 to 1.25 .

We first notice that the maximum value of $R_{(2-1) /(1-0)}$ is displaced $\sim 40^{\prime \prime}$ to the north of the $\mathrm{CO}$ emission peak for cloud $\mathrm{A}$, and exactly matches an indentation in the radio continuum emission in the remnant's shell, close to the eastern protrusion. On the other hand, the two maxima are coincident for cloud $\mathrm{B}$. Assuming that the $\mathrm{CO}$ emission is representative of the mass distribution, this coincidence suggests that the increase of $\mathrm{CO}$ in the excited rotational state $J=2$ is linked to the increase in density and temperature at the cloud's core. Moreover, the average value of $\leq 1$ for $R_{(2-1) /(1-0)}$ in cloud $\mathrm{B}$ is characteristic of dense molecular gas ready to form massive stars $\left(0.7 \leq R_{(2-1) /(1-0)} \leq 1\right.$; Hasegawa 1997).

As regards cloud $\mathrm{A}$, we have found that the maximum value of $R_{(2-1) /(1-0)}$ is 1.22 . As mentioned above, typical values of $R_{(2-1) /(1-0)}$ for SNRs interacting with molecular clouds are higher than 1. Based on this fact, together with the apparent lack of relationship between the $R_{(2-1) /(1-0)}$ peak and the core of cloud $\mathrm{A}$, and the coincidence between the former and a deformation in the radio shell of G18.8+0.3, we believe that the increase of $R_{(2-1) /(1-0)}$ at this location arises from molecular gas shocked by the supernova remnant.

\section{Summary and conclusions}

We have investigated the molecular gas properties in the direction of G349.7+0.2 and G18.8+0.3. The present ${ }^{12} \mathrm{CO} J=1-0$, 2-1 and 3-2 observations provide new evidence of the physical association between the SNRs and the surrounding molecular clouds in spite of the absence of substantial broadening in the observed line profiles.

For $\mathrm{G} 349.7+0.2$ the main results can be summarized as follows:

(a) The molecular cloud detected in the neighbourhood of G349.7+0.2 near $v_{\mathrm{LSR}} \sim+16 \mathrm{~km} \mathrm{~s}^{-1}$ has an average linear size of about $7 \mathrm{pc}$, a velocity dispersion of the order of $4 \mathrm{~km} \mathrm{~s}^{-1}$, a molecular mass of $\sim 10^{4} M_{\odot}$, and a volume density 
Table 3. Observed and derived parameters for the CO Clouds observed towards G18.8+0.3.

\begin{tabular}{lcc}
\hline \hline Parameters & Cloud A & Cloud B \\
\hline Center (RA, Dec J2000) & $18^{\mathrm{h}} 24^{\mathrm{m}} 13^{\mathrm{s}},-12^{\circ} 27^{\prime} 50^{\prime \prime}$ & $18^{\mathrm{h}} 24^{\mathrm{m}} 10^{\mathrm{s}},-12^{\circ} 29^{\prime} 30^{\prime \prime}$ \\
Angular size (') & 150 & 120 \\
$V_{\text {cent }}\left(\mathrm{km} \mathrm{s}^{-1}\right)$ & +19.9 & +19.7 \\
$\Delta v\left(\mathrm{~km} \mathrm{~s}^{-1}\right)$ & 7.5 & 4.8 \\
$T_{\text {peak }}^{B}(\mathrm{~K})$ & 15.3 & 12.4 \\
Total column density $\left(\mathrm{cm}^{-2}\right)$ & $2.5 \times 10^{22}$ & $1.8 \times 10^{22}$ \\
Molecular mass $\left(M_{\odot}\right)$ & $4.4 \times 10^{4}$ & $2.1 \times 10^{4}$ \\
Volume density $\left(\mathrm{cm}^{-3}\right)$ & $1.2 \times 10^{3}$ & $1.0 \times 10^{3}$ \\
\hline
\end{tabular}

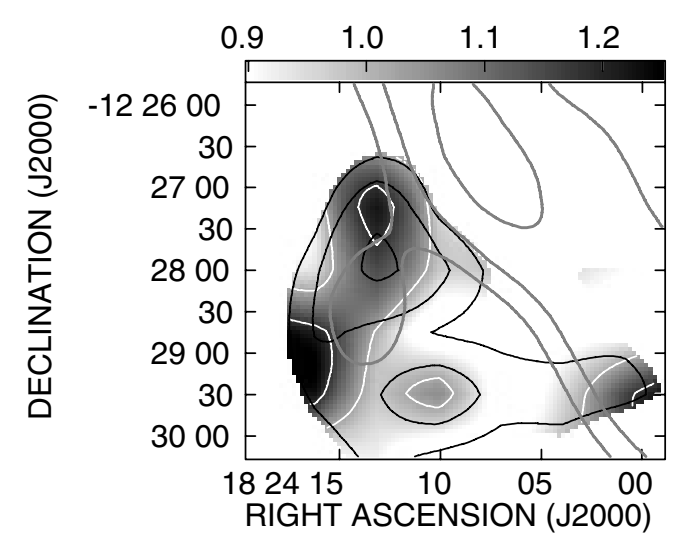

Fig. 13. Line ratios $R_{(2-1) /(1-0)}$ obtained towards the eastern side of G18.8+0.3, in greyscale and white contours. Black contours represent the CO $J=2-1$ emission, while grey thick lines correspond to the radio continuum. The intensity ratios vary between 0.9 (lighter) and 1.25 (darker). Pixels where the $\mathrm{CO} J=1-0$ emission was lower than $70 \mathrm{~K} \mathrm{~km} \mathrm{~s}^{-1}$, have been blanked.

of $\sim 10^{3} \mathrm{~cm}^{-3}$. The infrared color temperature of the associated dust, as derived from the $60 / 100 \mu \mathrm{m}$ ratio $\left(T_{\text {dust }}=37 \mathrm{~K}\right)$, is compatible with shock-heated dust. An additional indication of shock/cloud interaction is provided by the line ratios in the associated cloud. We have obtained $R_{2-1 / 1-0} \sim 1.5, R_{3-2 / 2-1} \sim$ 2.4 and $R_{3-2 / 1-0} \sim 3.5$. For typical quiescent molecular clouds, where the $J=2$ and $J=3$ levels are subthermally excited, the ratios are usually less than unity (e.g. $R_{3-2 / 2-1}=0.5$ for the Taurus complex, Falgarone et al. 1991).

At high velocities we have detected in the ${ }^{12} \mathrm{CO} J=1-0$ and $J=2-1$ lines an isolated cloud near $v \sim-109 \mathrm{~km} \mathrm{~s}^{-1}$ which overlaps the southern radio continuum and X-ray maxima. It is remarkable that this is the only molecular feature discovered (within the whole $\pm 1200 \mathrm{~km} \mathrm{~s}^{-1}$ velocity interval inspected) in positional coincidence with the brightest radio and X-ray feature in $\mathrm{G} 349.7+0.2$. However, a line ratio of only 0.5 makes a shocked gas origin for this feature unlikely. Besides, its association with a $22 \mathrm{kpc}$ distant $S N R$ is difficult to prove because the radial velocity of this cloud is close to the tangent point in this direction of the Galaxy.

(b) From the analysis of the total absorbing column density and its comparison with the X-ray results, we conclude that the associated molecular cloud is placed behind G349.7+0.2 in the direction of the line of sight. In this scenario the total absorbing gas between us and the SNR is $\sim 6.9 \times 10^{22} \mathrm{~cm}^{-2}$. Probably most of the molecular mass resides in smaller clumps, unresolved at the present angular resolution, that evaporate rapidly after the passage of the SN shock. The mass already evaporated would be responsible for the central X-ray emission. The denser material in the core of the cloud (as probed by the higher transitions) has not evaporated yet, but is experiencing the action of the SN shock, as revealed by the bright radio ridge that crosses the central part of G349.7+0.2, which perfectly matches the shape of the molecular cloud as traced by the higher transition lines.

(c) The analysis of the $J=2-1$ and $J=3-2$ lines shape around $v_{\mathrm{LSR}} \sim+16 \mathrm{~km} \mathrm{~s}^{-1}$ reveals substantial asymmetries. Near the center the spectra have a Gaussian shape, and the spectral asymmetry increases from the center to the borders of the cloud. Spectra on the eastern half of the cloud show a red wing, while spectra of the western half show a blue wing. We interpret the observed asymmetries as evidence of the kinematical effects induced by the shock/cloud interaction. The SN shock is running into the denser part of the molecular cloud and has probably begun to disrupt it, pushing the smaller clumps away from us on the eastern part, and toward us on the west side.

(d) The central velocities of the five associated $\mathrm{OH}$ $(1720 \mathrm{MHz})$ masers agree very well with the local $\mathrm{CO}$ peak velocity in three cases, while in the remaining two cases they agree with the radial velocity of a secondary, fainter component of the CO spectrum, which appears blended with the main one.

Theoretical studies suggest that $\mathrm{OH}(1720 \mathrm{MHz})$ masers are more efficiently pumped in hot, dense shocked molecular clouds when the shock propagates transverse to the line of sight, or forms a large angle $\left(\geq 80^{\circ}\right)$ with it. If this is the rule, then for the masers detected in G349.7+0.2 we are led to the conclusion that once the $\mathrm{SN}$ shock has overrun and penetrated the cloud, the shock front is distorted and the $\mathrm{OH}$ masers are excited at the sites where locally transverse shocks hit denser molecular clumps.

For the SNR G18.8+0.3, the new high-resolution observations have resolved the associated molecular cloud into two clumps, named cloud A and B. The positional matching between the shock front position as traced by the non-thermal radio emission and the northern molecular clump (cloud A) is striking, confirming the early suggestions by Dubner et al. (1999) of SNR/molecular cloud association. Based on the new study, and after correcting the distance to $\mathrm{G} 18.8+0.3(d=$ $14 \mathrm{kpc}$ ), we have refined the determination of the characteristic parameters of the associated gas. The molecular mass is estimated to be about $4.4 \times 10^{4} M_{\odot}$ and $1.2 \times 10^{4} M_{\odot}$ for clouds A 
and $\mathrm{B}$, respectively. The volume densities are $n_{\mathrm{A}} \sim 1200 \mathrm{~cm}^{-3}$ and $n_{\mathrm{B}} \sim 1000 \mathrm{~cm}^{-3}$. The velocity width of the associated gas is $\sim 7 \mathrm{~km} \mathrm{~s}^{-1}$. The analysis of the line ratios in this case revealed a maximum of $R_{2-1 / 1-0}=1.25$ at a position that exactly matches an indentation in the radio continuum emission in the remnant's shell. This fact together with the morphological agreement argues in favor of the existence of a physical interaction between the SNR G18.8+0.3 and the adjoining molecular cloud.

Acknowledgements. We are very grateful to the staff of SEST for the support received during the observations, especially to the former director Dr. L.-A. Nyman. This work was supported by the CONICET grant 2136/00 and UBACYT A055/04.

\section{References}

Arendt, R. G., Dwek, E., \& Leisawitz, D. 1992, ApJ, 400, 562

Arikawa, Y., Tatematsu, K., Sekimoto, Y., \& Takahashi, T. 1999, PASJ, 51, L7

Bloemen, J. B. G. M., Strong, A. W., Mayer-Hasselwander, H. A., et al. 1986, A\&A, 154, 25

Brogan, C. L., Frail, D. A., Goss, W. M., \& Troland, T. H. et al. 2000, ApJ, 537, 875

Cao, Y., Terebey, S., Prince, T. A., \& Beichman, C. A. 1997, ApJS, 111,387

Castelletti, G., Dubner, G., Golap, K., et al. 2003, AJ, 126, 2114

Caswell, J. L., Murray, J. D., Roger, R. S., Cole, D. J., \& Cooke, D. J. 1975, A\&A, 45, 239

Dickman, R. L. 1978, ApJS, 37, 407

Digel, S. W., Grenier, I. A., Heithausen, A., Hunter, S. D., \& Thaddeus, P. 1996, ApJ, 463, 609

Dubner, G., Giacani, E., Reynoso, E., Goss, W. M., Roth, M., \& Green, A. 1999, AJ, 118, 930

Dubner, G. M., Giacani, E. B., Goss, W. M., Moffett, D. A., \& Holdaway, M. 1996, AJ, 111, 1304
Elitzur, M. 1976, ApJ, 203, 124

Falgarone, E., Phillips, T. G., \& Walker, C. K. 1991, ApJ, 378, 186

Fich, M., Blitz, L., \& Stark, A. A. 1989, ApJ, 342, 272

Frail, D. A., Goss, W. M., Reynoso, E. M., Giacani, E. B., Green, A. J., \& Otrupcek, R. 1996, AJ, 111, 1651

Frail, D. A., \& Mitchell, G. F. 1998, ApJ, 508, 690

Green, A. J., Frail, D. A., Goss, W. M., \& Otrupcek, R. 1997, AJ, 114, 2058

Hasegawa, T. 1997, in CO: Twenty Five Years of Millimeter-Wave Spectroscopy, ed. W. B. Latter, S. J. E. Radford, P. R. Jewell, \& J. Bally (Dorchredt: Kluwer Academic Publishers), 69

Junkes, N., Fürst, E., \& Reich, W. 1992, A\&A, 261, 289

Koo, B., Rho, J., Reach, W. T., Jung, J., \& Mangum, J. G. 2001, ApJ, 552,175

Koralesky, B., Frail, D. A., Goss, W. M., Claussen, M. J., \& Green, A. J. 1998, AJ, 116, 1323

Lazendic, J. S., Wardle, M., Green, A. J., Whiteoak, J. B., \& Burton, M. G. 2002, in Neutron Stars in Supernova Remnants, ASP Conf. Ser., 271, 399

Lockett, P., Gauthier, E., \& Elitzur, M. 1999, ApJ, 511, 235

Reynoso, E. M., \& Mangum, J. G. 2000, ApJ, 545, 874

Reynoso, E. M., \& Mangum, J. G. 2001, AJ, 121, 347

Rho, J., \& Petre, R. 1998, ApJ, 503, L167

Seab, C. G., \& Shull, J. M. 1983, ApJ, 275, 652

Seta, M., Hasegawa, T., Dame, T. M., et al. 1998, ApJ, 505, 286

Shaver, P. A., Salter, C. J., Patnaik, A. R., van Gorkom, J. H., \& Hunt, G. C. 1985, Nature, 313, 113

Slane, P., Chen, Y., Lazendic, J. S., \& Hughes, J. P. 2002, ApJ, 580, 904

Stark, A. A., Gammie, C. F., Wilson, R. W., et al. 1992, ApJS, 79, 77

Tatematsu, K., Fukui, Y., Nakano, M., Kogure, T., Ogawa, H., \& Kawabata, K. 1987, A\&A, 184, 279

Wardle, M. 1999, ApJ, 525, L101

Yamauchi, S., Koyama, K., Kinugasa, K., et al. 1998, Astron. Nachr., 319, 111

Yusef-Zadeh, F., Wardle, M., Rho, J., \& Sakano, M. 2003, ApJ, 585, 319 